\title{
Differential equations for loop integrals without squared propagators
}

\author{
Jorrit Bosma \\ ETH Zürich, Wolfang-Pauli-Strasse 27, 8093 Zürich, Switzerland \\ E-mail: jbosma@itp.phys.ethz.ch
}

\section{Kasper J. Larsen*}

School of Physics and Astronomy, University of Southampton, Highfield, Southampton, SO17 1BJ, United Kingdom

E-mail: Kasper.Larsen@soton.ac.uk

\section{Yang Zhang}

ETH Zürich, Wolfang-Pauli-Strasse 27, 8093 Zürich, Switzerland

PRISMA Cluster of Excellence, Johannes Gutenberg University, 55128 Mainz, Germany

E-mail: zhang@uni-mainz.de

\begin{abstract}
We provide a sufficient condition for avoiding squared propagators in the intermediate stages of setting up differential equations for loop integrals. This condition is satisfied in a large class of two- and three-loop diagrams. For these diagrams, the differential equations can thus be computed using "unitarity-compatible" integration-by-parts reductions, which simplify the reduction problem by avoiding integrals with higher-power propagators.
\end{abstract}

Loops and Legs in Quantum Field Theory (LL2018)

29 April 2018 - 04 May 2018

St. Goar, Germany

\footnotetext{
* Speaker.
} 


\section{Introduction}

The physics program of the Large Hadron Collider (LHC) demands precision calculations of cross sections of Standard Model processes to attain a quantitative understanding of the background and in turn facilitate the extraction of new physics signals. The required accuracy is typically nextto-next-to leading order (NNLO) in fixed-order perturbation theory, in order to match the parton distribution uncertainties and the experimental precision. Out of the contributions to NNLO cross sections, the double-virtual one, i.e. the two-loop scattering amplitude, is the main bottleneck.

Calculation of multi-loop amplitudes proceeds in two stages. In the first step, the amplitude is rewritten as a linear combination of a basis of integrals through the use of discrete symmetries and integration-by-parts (IBP) reductions. The latter are linear relations among loop integrals which arise from the vanishing integration of total derivatives in dimensional regularization,

$$
\int \prod_{i=1}^{L} \frac{\mathrm{d}^{D} \ell_{i}}{\mathrm{i} \pi^{D / 2}} \sum_{j=1}^{L} \frac{\partial}{\partial \ell_{j}^{\mu}} \frac{v_{j}^{\mu} P}{D_{1}^{\alpha_{1}} \cdots D_{m}^{\alpha_{m}}}=0,
$$

where $P$ and the vectors $v_{j}^{\mu}$ are polynomial in the internal and external momenta, the $D_{k}$ denote inverse propagators, and the $\alpha_{i}$ are integers. Upon applying Gaussian elimination [1,2] to a suitably large system of IBP identities (1.1), one obtains the IBP reductions [3-16], which express the majority of the contributing loop integrals as linear combinations of a small basis of integrals.

In the second step, one sets up differential equations for the basis integrals [17-26]. Letting $x_{m}$ denote an external kinematical invariant, $\varepsilon=\frac{4-D}{2}$ the dimensional regulator, and $\mathscr{I}(x, \varepsilon)=$ $\left(\mathscr{I}_{1}(x, \varepsilon), \ldots, \mathscr{I}_{M}(x, \varepsilon)\right)$ the basis of integrals, we have the following first-order linear system,

$$
\frac{\partial}{\partial x_{m}} \mathscr{I}(x, \varepsilon)=A_{m}(x, \varepsilon) \mathscr{I}(x, \varepsilon),
$$

where in practice one uses the IBP reductions to decompose the derivatives $\frac{\partial \mathscr{I}_{j}}{\partial x_{m}}$ in the basis. With appropriate boundary conditions, eq. (1.2) can be solved to produce expressions for the basis integrals. This method has proven to be a powerful tool for computing multi-loop integrals.

The aim of these proceedings, based on ref. [27], is to investigate to what extent the IBP reduction formalisms of refs. [10-12,15] are compatible with differential equations of the type in eq. (1.2). The main idea of these IBP reduction formalisms is to choose the $v_{j}^{\mu}\left(\ell_{i}\right)$ in eq. (1.1) such that the resulting IBP identities do not involve squared propagators. The resulting IBP identities thus involve a more limited set of integrals and therefore produce significantly smaller linear systems to be solved. The question we wish to address is therefore whether it is possible to set up differential equations of the form (1.2) without generating integrals with squared propagators in intermediate stages.

\section{Differential equations in Baikov representation}

We begin by fixing our notation and conventions. We consider a Feynman integral with $L$ loops, $k$ propagators and $m-k$ irreducible scalar products (i.e., polynomials in the loop momenta 
and external momenta which cannot be expressed as a linear combination of the inverse propagators). We apply dimensional regularization and normalize the integral as follows,

$$
I\left(N ; \alpha_{1}, \ldots, \alpha_{m} ; D\right) \equiv \int \prod_{j=1}^{L} \frac{\mathrm{d}^{D} \ell_{j}}{\mathrm{i} \pi^{D / 2}} \frac{N}{D_{1}^{\alpha_{1}} \cdots D_{m}^{\alpha_{m}}} .
$$

Here $N$ denotes a polynomial in the linearly independent external momenta $p_{1}, \ldots, p_{E}$ and the loop momenta $\ell_{1}, \ldots, \ell_{L}$, and $m=L E+L(L+1) / 2$. The propagators are labeled such that,

$$
\begin{array}{ll}
\alpha_{i} \geq 1 & \text { for } \quad i=1, \ldots, k \\
\alpha_{i} \leq 0 & \text { for } \quad i=k+1, \ldots, m .
\end{array}
$$

We remark that eq. (2.1) does not give a unique representation, as $D_{k+1}^{-\alpha_{k+1}} \cdots D_{m}^{-\alpha_{m}}$ can be absorbed into $N$ to form a polynomial numerator, $I(N ; \alpha ; D)=I\left(N \prod_{j=k+1}^{m} D_{j}^{-\alpha_{j}} ;\left(\alpha_{1}, \ldots, \alpha_{k}, 0\right) ; D\right)$. Nevertheless, we find it more convenient to use this notation than to fix the rescaling invariance.

The question we wish to address is whether differential equations of the form (1.2) can be set up without introducing integrals with squared propagators. To this end it is convenient to make use of the Baikov representation [28] in which the integration variables are the inverse propagators and irreducible numerator insertions, $z_{\alpha}=D_{\alpha}$ with $1 \leq \alpha \leq m$. The associated Jacobian involves the Gram determinants $U=\operatorname{det}_{i, j=1, \ldots, E}\left(p_{i} \cdot p_{j}\right)$ and $F=\operatorname{det}_{i, j=1, \ldots, E+L}\left(v_{i} \cdot v_{j}\right)$ where $\left\{v_{1}, \ldots, v_{E+L}\right\} \equiv$ $\left\{p_{1}, \ldots, p_{E}, \ell_{1}, \ldots, \ell_{L}\right\}$. Using this notation, the integral in eq. (2.1) has the following Baikov representation (up to an irrelevant kinematics-independent prefactor),

$$
I(N ; \alpha ; D)=U^{\frac{E-D+1}{2}} \int \frac{\mathrm{d} z_{1} \cdots \mathrm{d} z_{m}}{z_{1}^{\alpha_{1}} \cdots z_{m}^{\alpha_{m}}} F(z)^{\frac{D-L-E-1}{2}} N(z) .
$$

To write down differential equations of the form (1.2), we let $\left(I_{1}, \ldots, I_{M}\right)$ denote a basis of integrals and differentiate the Baikov representation (2.3) with respect to an arbitrary external invariant $\chi$, yielding,

$$
\frac{\partial}{\partial \chi} I_{j}\left(N_{j} ; \alpha ; D\right)=\frac{E-D+1}{2 U} \frac{\partial U}{\partial \chi} I_{j}\left(N_{j} ; \alpha ; D\right)+\frac{D-L-E-1}{2} I_{j}\left(\frac{1}{F} \frac{\partial F}{\partial \chi} N_{j} ; \alpha ; D\right) .
$$

We observe that the $\frac{1}{F}$ factor in the second term effectively modifies the integration measure in eq. (2.3), shifting the space-time dimension from $D$ to $D-2$.

However, as proved in ref. [27], the second term of eq. (2.4) can always be expressed as a linear combination of $D$-dimensional integrals. This follows from the fact that there exist polynomials $\left(a_{1}, \ldots, a_{m}, b\right)$ in the $z_{\alpha}$ and the external kinematical invariants such that the following relation holds,

$$
\frac{\partial F}{\partial \chi}=\sum_{i=1}^{m} a_{i} \frac{\partial F}{\partial z_{i}}+b F
$$

referred to as fundamental ideal membership of $F$.

Using eq. (2.5) and integration by parts in each $z_{i}$, one finds that eq. (2.4) takes the form

$$
\frac{\partial}{\partial \chi} I_{j}\left(N_{j} ; \alpha ; D\right)=\frac{E-D+1}{2 U} \frac{\partial U}{\partial \chi} I_{j}\left(N_{j} ; \alpha ; D\right)+I_{j}\left(Q_{j} ; \alpha ; D\right),
$$


where the insertion $Q_{j}$ is given by,

$$
Q_{j}=\sum_{i=1}^{m}\left[\alpha_{i} \frac{a_{i} N_{j}}{z_{i}}-\frac{\partial}{\partial z_{i}}\left(a_{i} N_{j}\right)\right]+\frac{D-L-E-1}{2} b N_{j} .
$$

The resulting right-hand side of eq. (2.6) thus involves only $D$-dimensional integrals. Upon applying integration-by-parts reductions to the the right-hand sides for each $j=1, \ldots, M$, we find differential equations of the form (1.2).

\section{Differential equations without squared propagators}

Having set up the differential equations (1.2) in Baikov representation it is now straightforward to examine whether it is possible to avoid introducing integrals with squared propagators. From eqs. (2.6)-(2.7) we observe that terms with positive $\alpha_{i}$ will produce squared propagators for a generic polynomial $a_{i}$. However, provided it is possible to choose the polynomials $a_{i}$ such that,

$$
a_{i}=z_{i} b_{i} \quad \text { for } \quad i=1, \ldots, k,
$$

where $b_{i}$ denote polynomials, the insertion (2.7) takes the following form,

$$
Q_{j}=\sum_{i=1}^{k}\left[\left(\alpha_{i}-1\right) b_{i} N_{j}-z_{i} \frac{\partial\left(b_{i} N_{j}\right)}{\partial z_{i}}\right]+\sum_{i=k+1}^{m}\left[\alpha_{i} \frac{a_{i} N_{j}}{z_{i}}-\frac{\partial\left(a_{i} N_{j}\right)}{\partial z_{i}}\right]+\frac{D-L-E-1}{2} b N_{j} .
$$

We observe that the only occurrence of $\frac{1}{z_{i}}$ is within the sum over the range $k+1 \leq i \leq m$. However, in this range $\frac{1}{z_{i}}$ occurs in the integrands with non-positive $\alpha_{i}$, cf. eqs. (2.2) and (2.3). That is, the $\frac{1}{z_{i}}$ in eq. (3.2) can at most introduce a propagator, but never double a propagator already present.

We conclude that no integrals with squared propagators are generated in setting up differential equations of the form (1.2) provided that the following relation holds,

$$
\frac{\partial F}{\partial \chi}=\sum_{i=1}^{k} b_{i} z_{i} \frac{\partial F}{\partial z_{i}}+\sum_{i=k+1}^{m} a_{i} \frac{\partial F}{\partial z_{i}}+b F
$$

Eq. (3.3) can be rewritten as the equivalent statement

$$
\frac{\partial F}{\partial \chi} \in\left\langle z_{1} \frac{\partial F}{\partial z_{1}}, \ldots, z_{k} \frac{\partial F}{\partial z_{k}}, \frac{\partial F}{\partial z_{k+1}}, \ldots, \frac{\partial F}{\partial z_{m}}, F\right\rangle,
$$

which we refer to as enhanced ideal membership of $F$.

Ideal membership (3.4) can be determined by computing a Gröbner basis $\mathscr{G}$ of the ideal on the right-hand side and then computing the remainder $r$ of $\frac{\partial F}{\partial \chi}$ after polynomial division with respect to $\mathscr{G}$. Namely, eq. (3.4) holds if and only if $r=0$. Alternatively, one can solve explicitly for the cofactors $\left(b_{1}, \ldots, b_{k}, a_{k+1}, \ldots, a_{m}, b\right)$ by starting with Ansätze which are linear in $\left(z_{1}, \ldots, z_{m}\right)$ and iteratively allowing for cofactors of higher degree. This is an efficient approach in practice, as cofactors are typically of low degrees and thus lead to linear systems of manageable sizes.

The enhanced ideal membership turns out to hold for a large class of multi-loop integrals. Some examples are illustrated in figure 1. At the same time we note that the enhanced ideal membership (3.4) is not a general property of the Baikov polynomial $F$ : e.g., the diagram in figure 2 provides a counterexample. 

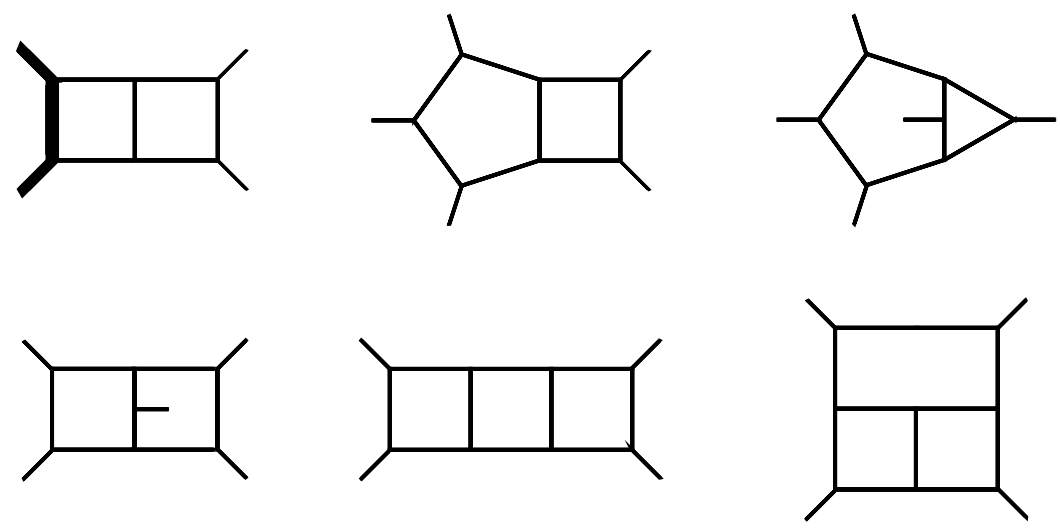

Figure 1: A selection of diagrams for which the enhanced ideal membership in eq. (3.3) holds. The bold lines represent massive momenta and propagators.

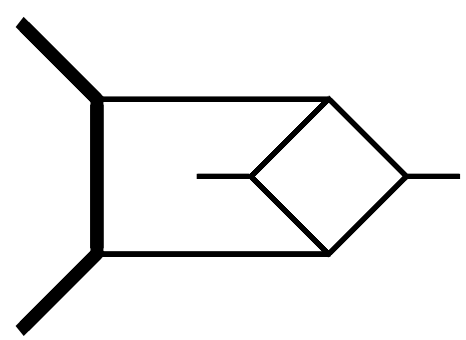

Figure 2: Non-planar double-box diagram. The bold lines represent massive momenta and propagators. For this diagram, the enhanced ideal membership (3.3) does not hold.

\section{Example}

As an application of the formalism in sections 2 and 3, let us work out the cofactors and the differential equations of the fully massless planar double-box diagram shown in figure 3 .

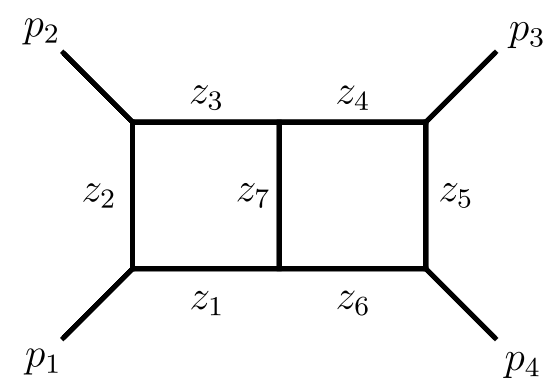

Figure 3: The fully massless planar double-box diagram. All external momenta are taken to be outgoing. 
In the case at hand we have $m=2 \cdot 3+2 \cdot 3 / 2=9$ Baikov variables which we define as follows,

$$
\begin{array}{lll}
z_{1}=\ell_{1}^{2}, & z_{2}=\left(\ell_{1}-p_{1}\right)^{2}, & z_{3}=\left(\ell_{1}-p_{1}-p_{2}\right)^{2}, \\
z_{4}=\left(\ell_{2}+p_{1}+p_{2}\right)^{2}, & z_{5}=\left(\ell_{2}-p_{4}\right)^{2}, & z_{6}=\ell_{2}^{2}, \\
z_{7}=\left(\ell_{1}+\ell_{2}\right)^{2}, & z_{8}=\left(\ell_{1}+p_{4}\right)^{2}, & z_{9}=\left(\ell_{2}+p_{1}\right)^{2} .
\end{array}
$$

We wish to set up differential equations for a basis of the vector space spanned by the diagram in figure 3 and its subdiagrams. An integral basis $\mathscr{I}$ can be obtained with AzURITE [29]. Setting $I_{\alpha} \equiv I(1 ; \alpha ; D)$, it finds

$$
\begin{aligned}
\mathscr{I}=\left(s^{-1+2 \varepsilon} I_{(0,1,0,0,1,0,1,0,0)},\right. & s^{-1+2 \varepsilon} I_{(1,0,0,1,0,0,1,0,0)}, \\
s^{2 \varepsilon} I_{(1,0,1,0,1,0,1,0,0)}, & s^{2 \varepsilon} I_{(1,0,1,1,0,1,0,0,0)}, \\
s^{1+2 \varepsilon} I_{(1,1,0,1,1,0,1,0,0)}, & \left.s^{1+2 \varepsilon} I_{(1,1,1,0,1,0,1,0,0)}, \quad s^{3+2 \varepsilon} I_{(1,1,1,1,1,1,1,0,0)}, \quad s^{2+2 \varepsilon} I_{(1,1,1,1,1,1,1,-1,0)}\right) .
\end{aligned}
$$

We rescaled the basis integrals $I_{\alpha}$ by $s^{|\alpha|-4+2 \varepsilon}$ to render them dimensionless. Thus, the integrals in eq. (4.2) depend on kinematics only through the dimensionless ratio $\chi \equiv t / s$.

We are therefore interested in differential equations for the basis integrals in eq. (4.2) taken with respect to $\chi$. In the case at hand, we find that the following property, slightly stronger than eq. (3.3), holds,

$$
\frac{\partial F}{\partial \chi}=\sum_{i=1}^{9} b_{i} z_{i} \frac{\partial F}{\partial z_{i}}+b F
$$

By writing Ansätze for $\left(b_{i}, b\right)$ which are linear in $\left(z_{1}, \ldots, z_{9}\right)$ and solving the resulting linear system, one finds the following cofactors, setting $\mathbf{b}=\left(b_{1}, \ldots, b_{9}\right)$,

$$
\begin{aligned}
& \mathbf{b}=\left(\frac{z_{3}-z_{8}}{\chi(\chi+1) s}, \frac{z_{3}-z_{8}-\chi s-s}{\chi(\chi+1) s}, \frac{z_{3}-z_{8}-s}{\chi(\chi+1) s}, \frac{z_{4}-z_{5}-s}{\chi(\chi+1) s}, \frac{z_{4}-z_{5}-s}{\chi(\chi+1) s}, \frac{z_{4}-z_{5}}{\chi(\chi+1) s}, \frac{z_{3}+z_{4}-z_{5}-z_{8}-s}{\chi(\chi+1) s}, \frac{z_{3}-z_{8}-s}{\chi(\chi+1) s}, \frac{z_{4}-z_{5}-\chi s-s}{\chi(\chi+1) s}\right) \\
& b=-\frac{2 z_{3}+2 z_{4}-2 z_{5}-2 z_{8}-2 \chi s-3 s}{\chi(\chi+1) s} .
\end{aligned}
$$

We insert these expressions into eqs. (3.1) and (3.2) and subsequently apply IBP reductions to the resulting right-hand sides to find a system of differential equations of the desired form,

$$
\frac{\partial}{\partial \chi} \mathscr{I}(\chi, \varepsilon)=A(\chi, \varepsilon) \mathscr{I}(\chi, \varepsilon) \text {. }
$$

The resulting coefficient matrix $A(\chi, \varepsilon)$ is not particularly illuminating. Rather than presenting its explicit form, we take one further step [22] and rotate to a new basis $\mathscr{J}(\chi, \varepsilon)$,

$$
\mathscr{J}(\chi, \varepsilon)=U(\chi, \varepsilon) \mathscr{I}(\chi, \varepsilon),
$$

in which the coefficient matrix becomes proportional to $\varepsilon$, whereby the system is in canonical form.

We can find a change-of-basis matrix $U$ with the desired property by using Fuchsia [30]. Providing the coefficient matrix $A(\chi, \varepsilon)$ computed in eq. (4.5) as input, it finds

$$
U=\operatorname{diag}\left(\frac{(1-2 \varepsilon)(1-3 \varepsilon)(2-3 \varepsilon)}{120 \varepsilon^{3} \chi}, \frac{(1-2 \varepsilon)(1-3 \varepsilon)(-2+3 \varepsilon)}{120 \varepsilon^{3}}, \frac{(1-2 \varepsilon)(1-3 \varepsilon)}{24 \varepsilon^{2}}, \frac{(1-2 \varepsilon)^{2}}{18 \varepsilon^{2}},-\frac{\chi+1}{2}, \frac{-1+2 \varepsilon}{6 \varepsilon},-\frac{\chi}{2}, \frac{1}{2}\right) .
$$


In the new basis $\mathscr{J}(\chi, \varepsilon)$, we have the explicit differential equations

$$
\frac{\partial}{\partial \chi} \mathscr{J}(\chi, \varepsilon)=\varepsilon\left(\frac{a_{0}}{\chi}+\frac{a_{-1}}{\chi+1}\right) \mathscr{J}(\chi, \varepsilon),
$$

where $a_{0}$ and $a_{-1}$ are matrices with integer entries,

$$
a_{0}=\left(\begin{array}{cccccccc}
-2 & 0 & 0 & 0 & 0 & 0 & 0 & 0 \\
0 & 0 & 0 & 0 & 0 & 0 & 0 & 0 \\
0 & 0 & 0 & 0 & 0 & 0 & 0 & 0 \\
0 & 0 & 0 & 0 & 0 & 0 & 0 & 0 \\
-60 & -60 & 0 & 0 & -2 & 0 & 0 & 0 \\
20 & 0 & -4 & 0 & 0 & -2 & 0 & 0 \\
-360 & 360 & 72 & 0 & 12 & 36 & -20 \\
540 & -360 & -90 & -9 & -18 & -36 & 1 & 1
\end{array}\right), \quad a_{-1}=\left(\begin{array}{cccccccc}
0 & 0 & 0 & 0 & 0 & 0 & 0 & 0 \\
0 & 0 & 0 & 0 & 0 & 0 & 0 & 0 \\
0 & 0 & 0 & 0 & 0 & 0 & 0 & 0 \\
0 & 0 & 0 & 0 & 0 & 0 & 0 & 0 \\
0 & 0 & 0 & 0 & 2 & 0 & 0 & 0 \\
-20 & 0 & 4 & 0 & 0 & 1 & 0 & 0 \\
360 & -720 & -36 & 18 & -12 & -36 & 2 & 2 \\
-540 & 360 & 90 & -9 & 18 & 36 & -1 & -1
\end{array}\right) .
$$

Thus we have derived differential equations of the type (1.2) for the basis integrals in eq. (4.2) and achieved a canonical form of the system without introducing integrals with squared propagators in intermediate stages.

\section{Conclusions}

Differential equations of the form (1.2) provide a powerful method for computing multi-loop integrals. In practice, setting up such equations for multi-scale integrals is non-trivial, as the step of expressing the derivatives of the integrals in the basis requires integration-by-parts (IBP) reductions which are computationally intensive to generate. Refs. [10-12,15] provide a simplified approach to IBP reductions where integrals with squared propagators are avoided in intermediate stages, thus producing significantly smaller linear systems to be solved.

In these proceedings, based on ref. [27], we have addressed the question whether it is possible to set up differential equations of the form (1.2) without introducing integrals with squared propagators in intermediate stages, so that the formalism of refs. $[10-12,15]$ can be applied.

We have shown that a sufficient condition is that the Baikov polynomial $F$ satisfies eq. (3.3). This condition holds for a large class of multi-loop diagrams, including highly non-trivial loop diagrams whose differential equations are not attainable with standard methods. A sample is illustrated in figure 1. At the same time, we have identified a counterexample to eq. (3.3), shown in figure 2. An interesting open problem is therefore to classify the diagrams for which the enhanced ideal membership property (3.3) holds. Another interesting problem is to find closed formulas for the cofactors in eq. (3.3).

\section{References}

[1] S. Laporta, Phys. Lett. B504, 188 (2001), hep-ph/0102032.

[2] S. Laporta, Int. J. Mod. Phys. A15, 5087 (2000), hep-ph/0102033.

[3] C. Anastasiou and A. Lazopoulos, JHEP 07, 046 (2004), hep-ph/0404258. 
[4] A. V. Smirnov, JHEP 10, 107 (2008), 0807.3243.

[5] A. V. Smirnov, Comput. Phys. Commun. 189, 182 (2014), 1408.2372.

[6] C. Studerus, Comput. Phys. Commun. 181, 1293 (2010), 0912.2546.

[7] A. von Manteuffel and C. Studerus, (2012), 1201.4330.

[8] R. N. Lee, (2012), 1212.2685.

[9] P. Maierhöfer, J. Usovitsch, and P. Uwer, Comput. Phys. Commun. 230, 99 (2018), 1705.05610 .

[10] J. Gluza, K. Kajda, and D. A. Kosower, Phys.Rev. D83, 045012 (2011), 1009.0472.

[11] H. Ita, Phys. Rev. D94, 116015 (2016), 1510.05626.

[12] K. J. Larsen and Y. Zhang, Phys. Rev. D93, 041701 (2016), 1511.01071.

[13] A. von Manteuffel and R. M. Schabinger, Phys. Lett. B744, 101 (2015), 1406.4513.

[14] Z. Bern, M. Enciso, H. Ita, and M. Zeng, (2017), 1709.06055.

[15] J. Böhm, A. Georgoudis, K. J. Larsen, H. Schönemann, and Y. Zhang, (2018), 1805.01873.

[16] H. A. Chawdhry, M. A. Lim, and A. Mitov, (2018), 1805.09182.

[17] A. V. Kotikov, Phys. Lett. B254, 158 (1991).

[18] A. V. Kotikov, Phys. Lett. B267, 123 (1991).

[19] Z. Bern, L. J. Dixon, and D. A. Kosower, Nucl. Phys. B412, 751 (1994), hep-ph/9306240.

[20] E. Remiddi, Nuovo Cim. A110, 1435 (1997), hep-th/9711188.

[21] T. Gehrmann and E. Remiddi, Nucl. Phys. B580, 485 (2000), hep-ph/9912329.

[22] J. M. Henn, Phys. Rev. Lett. 110, 251601 (2013), 1304.1806.

[23] C. G. Papadopoulos, JHEP 07, 088 (2014), 1401.6057.

[24] J. Ablinger et al., Comput. Phys. Commun. 202, 33 (2016), 1509.08324.

[25] H. Frellesvig and C. G. Papadopoulos, JHEP 04, 083 (2017), 1701.07356.

[26] M. Zeng, JHEP 06, 121 (2017), 1702.02355.

[27] J. Bosma, K. J. Larsen, and Y. Zhang, Phys. Rev. D97, 105014 (2018), 1712.03760.

[28] P. A. Baikov, Phys. Lett. B385, 404 (1996), hep-ph/9603267.

[29] A. Georgoudis, K. J. Larsen, and Y. Zhang, Comput. Phys. Commun. 221, 203 (2017), 1612.04252 .

[30] O. Gituliar and V. Magerya, Comput. Phys. Commun. 219, 329 (2017), 1701.04269. 